\title{
Efecto repelente del aceite de neem (Azadirachta indica A. Juss) contra zancudos (Anophelex spp) en lechones
}

\author{
Repellent effect of neem oil (Azadirachta indica A. Juss) against mosquitoes \\ (Anophelex spp) in piglets
Andrea Llanos Gonzáles', Héctor Sánchez Suárez ${ }^{1,4}$, Gloria Ochoa Mogollón², Tessy Peralta Ortiz ${ }^{3}$, Alberto Ordinola-Zapata ${ }^{3}$

\section{Resumen}

\begin{abstract}
El objetivo del estudio fue evaluar la eficacia repelente para zancudos (Anophelex spp) de tres dosis de aceite de neem (Azadirachta indica A. Juss) en cerdos bajo condiciones de campo. La investigación se realizó en una época del año de abundancia de zancudos en Tumbes, Perú. Se trabajó con 40 lechones recién destetados, 10 por tratamiento, mantenidos en jaulas bajo techo protegidas contra zancudos. Para la prueba de campo se expusieron, en forma aleatoria, cuatro lechones por día en jaulas externas sin protección al zancudo (un animal diferente por cada tratamiento por día) durante 14 días en un periodo de dos meses. El aceite de neem se extrajo con el equipo soxhlet y la fórmula incluyó 30\% de glicerina, 1, 2 o 3\% de aceite de neem (T1, T2, T3, respectivamente), y llevado al 100\% con alcohol farmacéutico. Se aplicó $50 \mathrm{ml} /$ lechón distribuido en todo el cuerpo. Las picaduras y el número de zancudos fueron evaluados en cuatro ocasiones de 15 minutos durante tres horas por día (06:15, 07:00, 07:45, 08:30) con intervalos de 30 minutos de protección a los lechones. Los zancudos que picaban a los lechones (abdomen rojo) fueron colectados mediante aspiradoras manuales. El rendimiento de aceite de neem fue de $43.25 \pm 1.28 \%$. El porcentaje de repelencia fue de $88.74 \pm$ $6.15,75.66 \pm 11.8$ y $52.27 \pm 12.8 \%$ para $\mathrm{T} 1, \mathrm{~T} 2$ y T3, respectivamente $(\mathrm{p}<0.05)$. El porcentaje de protección (eficiencia del repelente) fue de $93.92 \pm 11.19,85.71 \pm 7.54$ y $67.78 \pm 3.66 \%$ para T1, T2 y T3, respectivamente $(\mathrm{p}<0.05)$. El número promedio de picaduras de zancudo en el grupo testigo T0 ( $\sin$ dosis de repelente) fue de $365.54 \pm 646.82$ picaduras/día, siendo diferente $(\mathrm{p}<0.05)$ a los grupos tratados T1, T2 y T3 $(49.98 \pm 89.11 ; 113.82 \pm 200.95$; $19.52 \pm 34.41$ picaduras/día, respectivamente, y sin diferencia significativa entre los gru-
\end{abstract}

${ }^{1}$ Escuela de Medicina Veterinaria y Zootecnia, Facultad de Ciencias Agrarias, Universidad Nacional de Tumbes, Perú

${ }^{2}$ Departamento de Biología y Bioquímica, Facultad de Ciencias de la Salud, Universidad Nacional de Tumbes Perú

${ }^{3}$ Facultad de Ingeniería Pesquera y Ciencias del Mar, Universidad Nacional de Tumbes, Perú

${ }^{4}$ E-mail: hsanchezs@untumbes.edu.pe

Recibido: 18 de marzo de 2019

Aceptado para publicación: 10 de enero de 2020

Publicado: 31 de marzo de 2020 
pos tratados. El Índice de Repelencia (IR) para T1, T2 y T3 fue de 0.47; 0.24 y 0.10, respectivamente, lo cual demuestra que el repelente utilizado en $\mathrm{T} 3$ mostró mayor nivel de repelencia y eficacia contra los zancudos.

Palabras clave: aceite de neem, picaduras, repelente para cerdos, eficiencia de repelente, repelentes vegetales

\section{AbSTRACT}

The aim of this study was to evaluate the repellent efficacy for mosquitoes (Anophelex spp) of three doses of neem oil (Azadirachta indica A. Juss) in pigs under field conditions. The research was carried out at a time of year of abundance of mosquitoes in Tumbes, Peru. Forty recently weaned piglets were used, 10 per treatment, kept in indoor cages protected against mosquitoes. For the field test, four piglets per day were exposed randomly in external cages without protection to the mosquito (a different animal for each treatment per day) for 14 days over a period of two months. Neem oil was extracted with the soxhlet apparatus and the formula included 30\% glycerin, 1,2 or 3\% neem oil (T1, T2, $\mathrm{T} 3$, respectively), and taken to $100 \%$ with pharmaceutical alcohol. A dose of $50 \mathrm{ml} /$ piglet distributed throughout the body was applied. The bites and the number of mosquitoes were evaluated on four occasions of 15 minutes for three hours per day $(06: 15,07: 00$, 07:45, 08:30) with intervals of 30 minutes of protection to the piglets. The mosquitoes biting the piglets (red abdomen) were collected using manual vacuum cleaners. The neem oil yield was $43.25 \pm 1.28 \%$. The percentage of repellency was $88.74 \pm 6.15,75.66 \pm 11.08$ and $52.27 \pm 12.83 \%$ for $\mathrm{T} 1, \mathrm{~T} 2$ and $\mathrm{T} 3$, respectively $(\mathrm{p}<0.05)$. The percentage of protection (repellent efficiency) was $93.92 \pm 11.19,85.71 \pm 7.54$ and $67.78 \pm 3.66 \%$ for $\mathrm{T} 1$, T2 and T3, respectively $(\mathrm{p}<0.05)$. The average number of mosquitos in the control group, T0 (without repellent dose) was $365.54 \pm 646.82$ bites/day, being different $(p<0.05)$ to the treated groups T1, T2 and T3 (49.98 $\pm 89.11 ; 113.82 \pm 299.95 ; 19.52 \pm 34.41$ bites/day, respectively, and without significant difference between the treated groups. The Repellency Index (IR) for T1, T2 and T3 was $0.47,0.24$ and 0.10 , respectively, which shows that the dose used in T3 showed a higher level of repellency and efficacy against mosquitoes.

Key words: neem oil, stings, repellent for pigs, repellent efficiency, plant repellents

\section{INTRODUCCIÓN}

Las áreas urbanas, suburbanas y cerca de cultivos están ligadas a la proliferación de zancudos, considerados como vectores de enfermedades y causante de laceraciones en la piel (Cachago y Ligña, 2018). A. aegypti es una especie antropofílica. El mosquito Anopheles se adapta a todo tipo de climas y se le encuentra en la región de Tumbes, Perú (Castro, 2015; Alemán et al., 2016). En los arrozales de la zona se encuentra Aedes spp, Anopheles spp y Culex spp (Chin, 2001; Duong et al., 2017). Las hembras del mosquito Anopheles pueden vivir hasta un mes (Astaiza et al., 1988). Su presencia está ligada a su ciclo de vida, ya que se mueven solo dentro de unos metros de distancia de su lugar de cría original (Morán, 2018). Los mosquitos antropofílicos localizan a sus presas mediante el calor corporal y el dióxido de carbono emanado por ellos (Olaya y Méndez, 2003). 
Los cerdos presentan la misma susceptibilidad que el humano ante la picadura de estos insectos (Barcomes, 2010), pudiendo causar pérdidas económicas a los productores por mermas en la producción e incluso muerte de lechones (Vargas, 1998; Morgan, 2012). Así mismo, el virus de la encefalitis japonesa B (E J) se transmite entre las aves, cerdos y humanos (Chin, 2001; Duong et al., 2017), causando pérdidas debidas a camadas anormales (Martínez y Juanes, 2006).

Los repelentes de insectos se utilizan para evitar el acercamiento de insectos a la piel (Lorrén et al., 2015). Algunas sustancias naturales o sintéticas poseen esta acción y son llamadas repelentes, aunque estas no matan (Egunyomi et al., 2010; Roldán et al., 2015). El ácido láctico y el dióxido de carbono, presente en el sudor de animales de sangre caliente, son sustancias atractivas para los mosquitos y es detectado por el receptor olfativo de las antenas que contienen los quimiorreceptores (Naseem et al., 2016). Los repelentes perturban la capacidad de los receptores en las antenas de los mosquitos para responder a la sede de estímulos (Olaya y Méndez, 2003).

Al aplicar el repelente, los solventes se evaporan, dejando el ingrediente activo sobre la piel (Castro-Gutiérrez y Diaz-Madrigal, 1998). La tendencia actual en en el uso de insecticidas y repelentes es hacia los productos de origen natural al considerarlos menos contaminantes, además de ser de fácil elaboración (Escalante et al., 2004; Hurtado et al., 2016). El repelente más usado para mosquitos en humanos y animales es el $\mathrm{N}$, $\mathrm{N}$-dietil-3-metilbenzamida (DEET), de gran efectividad, pero con residuos tóxicos (Morales, 2019), por lo que se investigan repelentes de origen vegetal para su reemplazo (Olaya y Méndez, 2003).

La planta de neem (Azadirachta indica A. Juss) tiene propiedades insecticidas, controla plagas de campo y almacén, y tiene uso medicinal, forestal y farmacológico (Novo et al., 1997; Celis et al., 2009; Fernández et al., 2009; Delgado et al., 2012; García, 2018). El azadiractin es el principio activo del neem, estructuralmente similar a la hormona ecdisona (hormona de la muda) de los insectos (Romero y Vargas, 2005; Fernández et al., 2009), y no presenta efectos secundarios y sus propiedades anti-inflamatorias son un buen complemento como repelente natural (Alemán et al., 2016).

Mayormente el neem se utiliza como extracto oleoso, el cual contiene la azadirachtina, compuesto muy conocido por su acción biológica (Vega-Jarquín, 2016). La proporción de este compuesto en el aceite de neem varía dependiendo del método de extracción y de la calidad de las semillas. La extracción con hexano resulta en el 35-44\% de aceite (Antón et al., 2016). El objetivo del presente estudio fue evaluar la eficacia repelente para zancudos de tres dosis de aceite de neem en cerdos, obtenidos bajo condiciones de campo.

\section{Materiales y MéTodos}

\section{Lugar del Estudio}

El estudio se llevó a cabo en la Facultad de Ciencias Agrarias (FCA) de la Universidad Nacional de Tumbes - UNT (Tumbes, Perú), adyacente a una zona arrocera, considerada como una zona de considerable proliferación de zancudos.

\section{Aceite de Semilla de Neem}

La semilla de neem (Azadirachta indica A. Juss) fue obtenida en la FCA-UNT. Se dejó secar por 15 días al medio ambiente, se separó la semilla de la cáscara $(2 \mathrm{~kg})$ y se realizó la molienda con un molino casero de tornillo sin fin. La extracción del aceite se realizó utilizando un equipo soxhlet empleando como solvente hexano (temperatura entre 80 y $90{ }^{\circ} \mathrm{C}$ ) (Tesfaye y Tefera, 2017; Shooshtari et al., 2013; Cárdenas, 2017). El rendimiento se calculó según fórmula del ex- 
tracto oleoso: $\mathrm{EE}=$ peso del aceite / peso de la muestra inicial (Esparza-Díaz et al., 2010).

\section{Repelente}

El repelente fue preparado siguiendo la metodología de Olaya y Méndez (2003), para lo cual se mezcló $1,2 \%$ o $3 \%$ del aceite de neem con $30 \%$ de glicerina, agregando alcohol etílico (96\%) hasta llegar al 100\% (T1: 130-69; T2: 2-30-68; T3: 3-30-67).

\section{Animales e Instalaciones}

Se adquirieron 40 lechones en una granja de crianza semi-intensiva del caserío El Limón (Tumbes, Perú), con peso vivo entre 10 y $20 \mathrm{~kg}$. Los animales se encontraban aparentemente saludables, libres de parásitos y de deformaciones congénitas.

Los lechones fueron estabulados en corrales con techo de eternit y con separaciones de malla metálica. Se utilizaron cuatro jaulas internas protegidas contra zancudos, de $4.5 \mathrm{~m}^{2}$ de área $($ de $3 \times 1.5 \times 1 \mathrm{~m})$ por tratamiento, que se encontraban dentro de corrales con techo de eternit y con separaciones de malla metálica. Las cuales fueron desinfectadas y fumigadas antes del inicio del experimento. Se colocaron 10 animales por jaula. Además, se utilizaron cuatro jaulas de malla metálica, sin protección para zancudos, ubicadas al exterior de los corrales, con un área de $1 \mathrm{~m}^{2}$ y una altura de $0.5 \mathrm{~m}$. Se colocó un animal por jaula.

\section{Evaluación de los lechones}

Los lechones fueron escogidos de camadas similares, y se distribuyeron en cuatro grupos, de acuerdo con su peso vivo: grupo 1, 10.0-11.9 kg; grupo 2: 12.0-13.9 kg; grupo 3: 14.0-15.9 kg; grupo 4: $>16 \mathrm{~kg}$ a más. Para formar tratamientos homogéneos, se seleccionó un lechón de cada grupo formando cuatro tratamientos (10 lechones por tratamiento colocados en una jaula interna).
Los lechones se lavaron con un jabón sin fragancia y agua fría el día previo al inicio de la prueba. Esta se realizó desde las 06:00 hasta las 08:45, periodo de mayor actividad de picadura de los zancudos en el área de estudio.

Para la prueba de repelencia en campo, cada día se escogió un lechón diferente entre los 10 de cada tratamiento y se les llevó a las jaulas externas (cuatro jaulas individuales, una por tratamiento) para ser expuestos a las picaduras de los zancudos. Las jaulas estaban distantes entre sí por $4 \mathrm{~m}$. La posición de los lechones en las jaulas externas fue rotada aleatoriamente en cada día de evaluación. Se utilizaron los métodos de ensayo disponibles y modificados para animales por la Organización Mundial de la Salud para cuantificar la protección y la duración del efecto de formulaciones repelentes en campo (WHO, 2009).

A cada uno de los lechones en las jaulas externas se pulverizó $50 \mathrm{ml}$ del repelente correspondiente al grupo experimental. El repelente fue aplicado sobre toda la superficie corporal del lechón. El grupo control (T0) no recibió aplicación alguna (Santamaría et al., 2012).

El experimento de repelencia se realizó durante 14 días en un periodo de 5 semanas (tres días consecutivos por semana-jueves, viernes y sábado) (Santamaría et al., 2012). En cada día se evaluó el efecto repelente por cuatro veces $(06: 15,07: 00$. 07:45, 08:30) con una duración de 15 minutos por cada evaluación. Los lechones tuvieron 30 minutos de descanso y protección entre evaluaciones, donde fue retirado de la jaula externa y devuelto a la jaula interna protegida. Los zancudos que picaron a los lechones fueron recolectados con cuatro aspiradores manuales a batería (uno por cada tratamiento) y llevados al laboratorio para el conteo respectivo; adicionalmente se tomó fotografías de los zancudos al momento en que picaban a los 
lechones, considerando como picadura al zancudo con abdomen dilatado de color rojo (Santamaría et al., 2012).

\section{Parámetros de Repelencia}

El efecto repelente fue evaluado mediante los siguientes parámetros:

- Porcentaje de repelencia (PRp). Se estimó según la fórmula indicada por Olaya y Méndez 2003): \%PRp = [(Ncp - Ntp) / (Ncp + Ntp)] x 100\%, donde \%PRp es el porcentaje de repelencia, Ncp es el número de mosquitos que picaron a los lechones a los que no se les aplicó el repelente, Ntp es el número de mosquitos que picaron a los lechones a los que sí se les aplicó el repelente.

- Porcentaje de protección (PP) o de eficiencia del repelente. Se determinó mediante el cálculo del número de mosquitos que pican a lechones con tratamiento comparado con el que no tiene tratamiento (Thorsell et al., 1989; WHO, 2009): $\mathrm{PP}=[(\mathrm{Nc}-\mathrm{Nt}) / \mathrm{Nc}] \times 100$, donde $\mathrm{Nc}=$ número de picaduras en el lechón testigo en determinado periodo de tiempo, $\mathrm{Nt}=$ número de picaduras en el lechón tratado en el mismo periodo

- Número de picaduras/día. Se midió por conteo directo de las ronchas dejadas por los zancudos en la piel de los lechones

- Índice de repelencia (IR). Se utilizó la fórmula adaptada por Sanabria y Ramírez (2013): $\mathrm{IR}=2 \mathrm{G} /(\mathrm{G}+\mathrm{P})$, donde IR es el índice de repelencia, $G$ es el porcentaje de zancudos en los lechones a los que se les aplicó el repelente, $\mathrm{P}$ es el porcentaje de zancudos en los lechones en los que no se aplicó el repelente. Para la interpretación del IR se consideró que para el $\mathrm{IR}=1$ el repelente no tuvo efecto; IR $>1$ el repelente atrajo a los zancudos; IR $<1$ el tratamiento repelió a los zancudos.

- Porcentaje de reducción de repelencia en función al tiempo de aplicación. Se- determinó según la fórmula descrita por Santamaría et al. (2012), WHO (2009) y Thorsell et al. (1989): \% de reducción de repelencia $=100[1-(\mathrm{Nt} / \mathrm{Nc})]$, donde $\mathrm{Nc}$ es el número de picaduras en el lechón testigo en un determinado periodo de tiempo, Nt es el número de picaduras en el lechón tratado en el mismo periodo de tiempo ( 3 horas en cuatro intervalos: entre 15 y 30,60 y 76,95 y 120,150 y 175 minutos).

Los resultados fueron analizados mediante análisis de varianza y la prueba de Duncan. La significancia estadística se fijó en $\mathrm{p}<0.05$.

\section{Resultados}

La extracción de aceite de neen mediante el método soxhlet fue de $43.25 \pm$ $1.28 \%$; rendimiento semejante al $40 \%$ reportado por Pérez et al. (2009).

El porcentaje de repelencia fue superior en el tratamiento $\mathrm{T} 3(88.74 \pm 6.15 \%)$, seguido del T2 con $75.66 \pm 11.08 \%$ y T 1 con $52.27 \pm 12.83 \%$, encontrándose diferencias significativas $(\mathrm{p}<0.05)$ entre grupos (Figura 1a). El porcentaje de protección (PP) o de eficiencia del repelente fue igualmente superior en T3 (93.92 $\pm 11.19 \%)$, seguido del T2 con $85.71 \pm 7.54 \%$ y $\mathrm{T} 1$ con $67.78 \pm 3.66 \%$, observándose diferencias significativas $(p<0.05)$ entre grupos (Figura $1 b)$.

El número promedio de picaduras de zancudo en el grupo testigo T0 ( $\sin$ dosis de repelente) fue de $365.54 \pm 646.82$ picaduras/ día), siendo significativamente diferente $(\mathrm{p}<0.05)$ de los grupos tratados T1, T2 y T3 $(49.98 \pm 89.11 ; 113.82 \pm 200.95 ; 19.52 \pm 34.41$ picaduras/día, respectivamente), y sin diferencia significativa entre los grupos tratados (Figura 1c). 

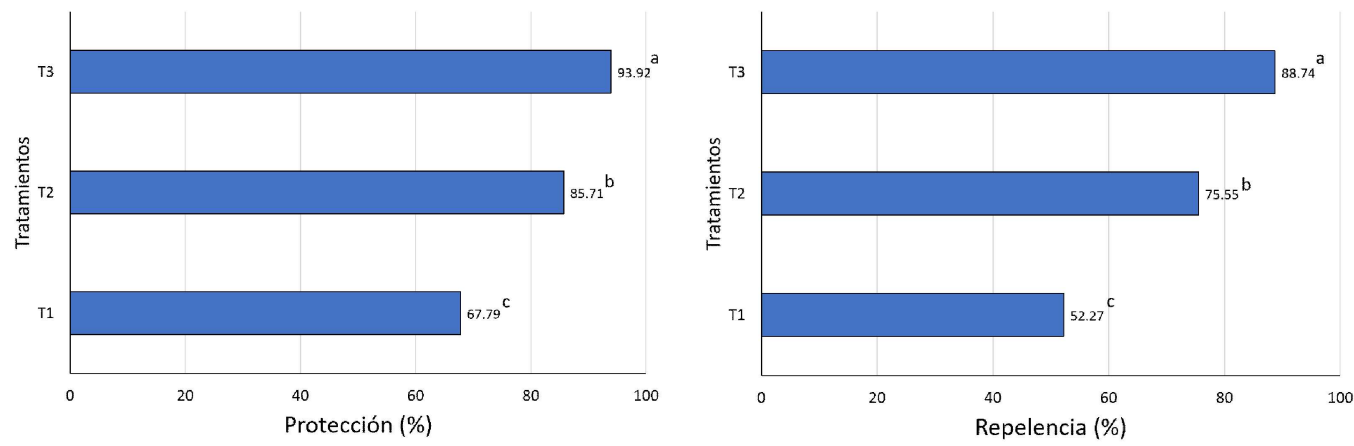

(a)

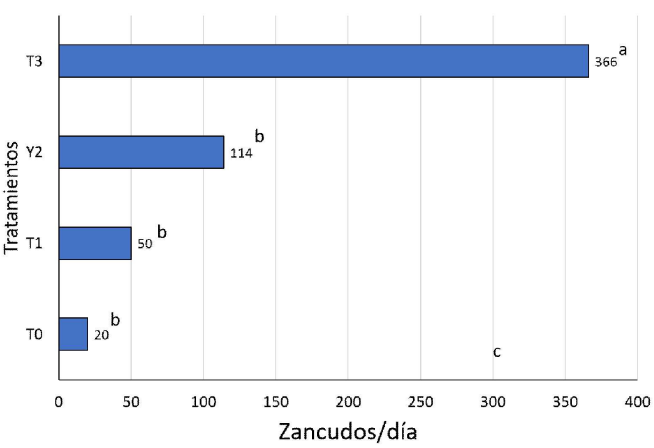

(b)

Figura 1. Porcentajes de repelencia y de protección, y número de picaduras de zancudos en lechones entre tratamientos. (10 lechones por grupo fueron rociados con un repelente conteniendo 1, 2 y $3 \%$ de aceite de neem (Azadirachta indica A. Juss) en T1, T2 y $\mathrm{T} 3$, respectivamente.

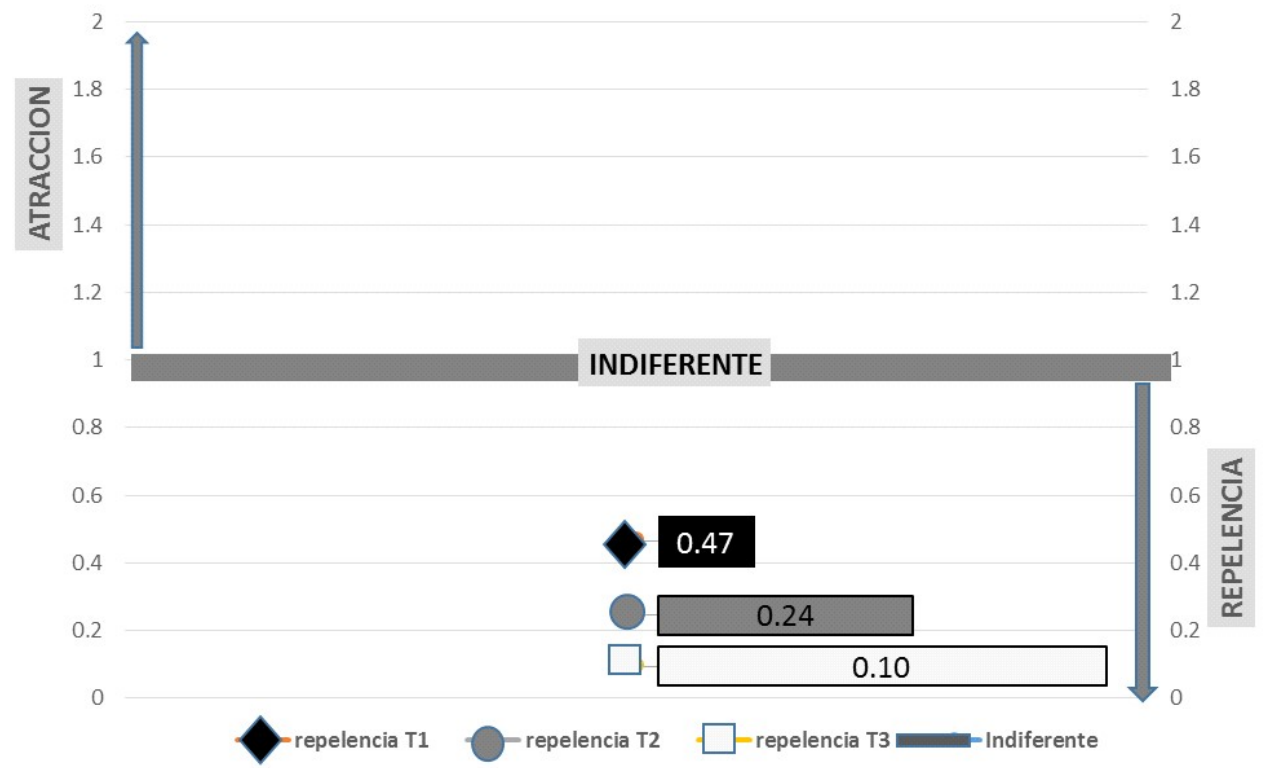

Figura 2. Índice de repelencia (comparación de la línea de atracción y repelencia) de repelentes conteniendo 1, 2 y $3 \%$ de aceite de neem (Azadirachta indica A. Juss) en T1, T2 y $\mathrm{T} 3$, respectivamente 


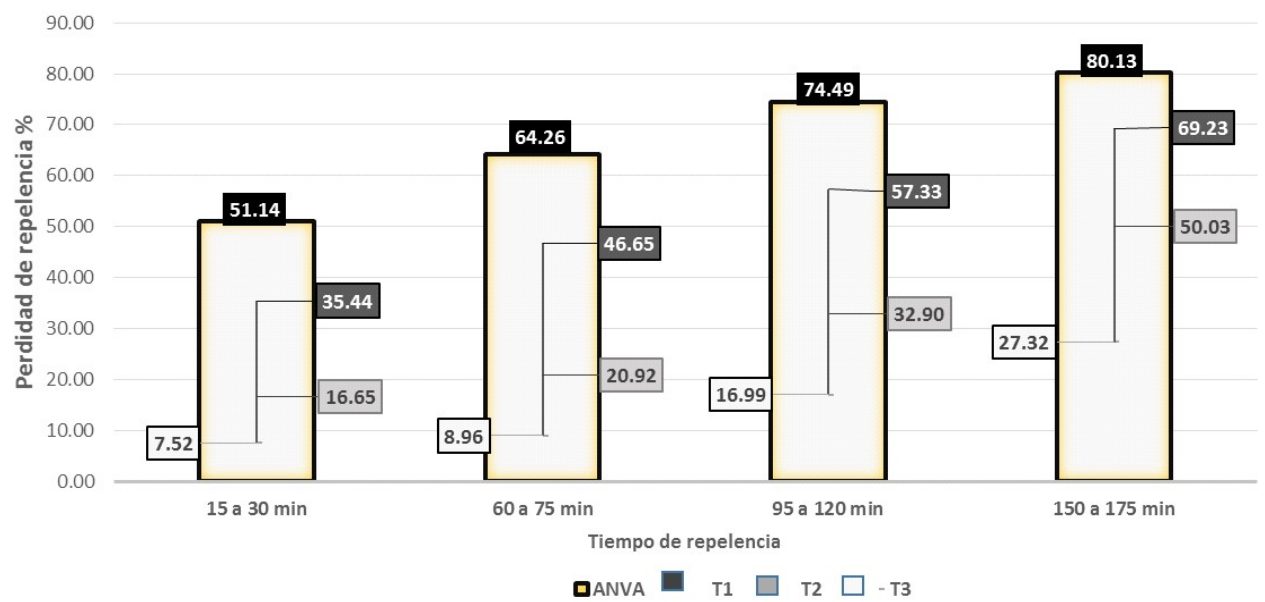

Figura 3. Porcentaje de la reducción de la repelencia total dentro de las tres horas de aplicado el repelente conteniendo 1,2 y $3 \%$ de aceite de neem (Azadirachta indica A. Juss) en $\mathrm{T} 1, \mathrm{~T} 2$ y $\mathrm{T} 3$, respectivamente

El Índice de Repelencia (IR) para T1, T2 y T3 fue de $0.47 ; 0.24$ y 0.10 , respectivamente (Figura 2), lo cual demuestra que el repelente utilizado en T3 mostró mayor nivel de repelencia a los zancudos.

El porcentaje de reducción de la repelencia, valor que está relacionado con la pérdida del efecto repelente en función al tiempo mostrado en la Figura 3, donde la disminución promedio total de la repelencia a los zancudos fue de $80.13 \pm 16.54 \%$ a los 175 minutos. En el caso de los tratamientos, la mayor pérdida fue en T1 $(69.23 \pm 4.11 \%)$, seguido de T2 (50.03 $\pm 4.47 \%)$ y T3 $(27.32 \pm$ $3.56 \%$ ) en dicho lapso.

\section{Discusión}

Se calcula la existencia de cerca de 85 especies de plantas con potenciales propiedades de repelencia contra insectos en Latinoamérica (Ramírez-Suárez et al., 2018), con componentes específicos (Alcalá-Orozco et al., 2017). Entre estas especies, la planta neem, considerada como un producto botánico bioactivo (Hernández-Castro et al., 2005), se presenta como una alternativa (Daza y Florez, 2006), habiendo sido utilizada como repelente de insectos en plantas, contra zancudos en humanos, como garrapaticida y contra moscas (Gruber, 1992; Esparza-Díaz et al., 2010; Ramírez-Suárez et al., 2018).

Los resultados obtenidos en la extracción de aceite de neem son semejantes a quienes recomiendan el método soxhlet utilizando hexano (Rodríguez et al., 2012; Tesfaye y Tefera, 2017). No obstante, existen métodos que utilizan maquinaria industrial y procesos fisicoquímicos con rendimiento de $76 \%$ y con máquinas de pelletsextrusado prensado con rendimientos de $64 \%$, pero cuyos procesos son de mayor costo (Escalante et al., 2004; Delgado et al., 2012; Cárdenas, 2017).

El neem posee un olor característico y que es considerado como la base del efecto repelente (Alemán et al., 2016), pudiendo interferir con el sentido del olfato del zancudo (Mindiola, 2019), por lo que la acción repelente se incrementa con que el aumento de la concentración del aceite de la planta. En trabajos de repelencia para humanos se utilizaron dosis entre 0.63 y $2.5 \%$ (López, 2015; 
Lorrén et al., 2015), pero concentraciones entre 3 y $5 \%$ son menos eficientes en hembras Aedes aegypti (Alemán et al., 2016). Como repelente para plantas se utilizan dosis de hasta 10\% (Novo et al., 1997; Chio y Yang, 2008; López, 2015; Boito et al., 2018).

Los resultados de repelencia y protección del presente estudio en lechones fueron similares a los obtenidos para mosquitos en humanos, donde se obtuvieron repelencias desde $57 \%$ hasta $94 \%$, dependiendo de la concentración de aceite de neem (Gruber, 1992; Daza y Florez, 2006; Regnault-Roger, 2012; Lorrén et al., 2015; Beltrán, 2018; Ramírez-Suárez et al., 2018). Así mismo, mostró efectos repelentes similares a compuestos de otras plantas como aceite de resino (Roldán et al., 2015) Melia azedarach L (Kebede et al., 2010), Elettaria cardamomum, Salvia officinalis y Lippia origanoides (Alcala-Orozco et al., 2019).

El neem mejora su efecto repelente en combinación con otros productos botánicos. Concentraciones de 2 y $5 \%$ de aceite de neem con aceite de coco en humanos contra Phlebotomus orientalis (vector de leishmaniasis visceral) dieron 96.3 y $98.3 \%$ de protección (Chio y Yang, 2008). Efectos similares se reportan con el extracto etanólico de la hoja (neem y Cinnamomum camphora) (Tabassum et al., 2018), neem y chinaberry (Melia azedarach L) (Kebede et al., 2010).

Debido a que la duración de la repelencia disminuye con el tiempo, se consideran las tres primeras horas como apropiadas para evaluar la acción repelente (Mandal, 2011). La disminución de repelencia en este trabajo fue mayor a la reportada utilizando neem y Cinnamomum camphora, donde solo disminuyó en $8 \%$ en una hora y $10 \%$ en la tercera hora (Tabassum et al., 2018). Así mismo, Martínez et al. (2016) usando sauco obtuvo mayor repelencia a las tres horas, resultado semejante a la dosis de T3 del presente experimento. Por otro lado, el aceite de neem con aceite de coco en humanos brindó una protección completa durante $12 \mathrm{~h}$ con- tra las picaduras de todas las especies de anophelidos (Kebede et al., 2010).

\section{Conclusiones}

- Las tres mezclas empleadas como repelentes contra zancudos tuvieron efecto positivo y significativo, con una tendencia no significativa de mayor porcentaje de repelencia $(88.74 \%)$ con el tratamiento con $3 \%$ de aceite de neem (Azadirachta indica A. Juss).

- La repelencia fue efectiva por lo menos por tres horas, siendo el tratamiento con $3 \%$ de aceite de neem quién presentó la menor disminución repelente $(21.41 \%)$.

\section{Literatura Citada}

1. Alcala-Orozco M, Caballero-Gallardo K, Stashenko EE, Olivero-Verbel J. 2019. Repellent and fumigant actions of the essential oils from Elettaria cardamomum (L) Maton, Salvia officinalis (L) Linnaeus, and Lippia origanoides (V) Kunth against Tribolium castaneum and Ulomoides dermestoides. J Essent Oil Bear P1 22: 18-18-30. doi: 10.1080/0972060X.2019.1585966

2. Alemán N, Gurdián X, Ortiz H. 2016. Evaluación in vivo de la actividad repelente de semillas Azadirachtaindica A. Juss (NEEM) contra Aedes aegypti vector de importancia en Salud Pública laboratorio de entomología MédicaMINSA Julio-Diciembre 2015. Tesis de Químico Farmacéutico. Managua, Nicaragua: Univ. Nacional Autónoma de Nicaragua. $22 \mathrm{p}$.

3. Antón R, Campos F, Guevara $S$, Guillén A, Ipanaqué M, León $R$. 2016. Diseño de la línea de producción de tres bioinsecticidas a base de la semilla, cáscara y residuos del grano en la extracción del aceite del árbol Azadiratcha indica. [Internet]. Dispo- 
nible en: https://pirhua.udep.edu.pe/ bitstream/handle/11042/2335/ 9._PYT_Informe_Final_Bioinsecticidas.-pdf? sequence $=1 \&$ isAllowed $=y$

4. Astaiza V, Murillo B, Fajardo O. 1988. Biología de Anopheles (Kerteszia) neivai H., D. \& K., 1913. (Diptera: Culicidae) en la costa Pacífica de Colombia: II. Fluctuación de la población adulta. Rev Saude Publ 22: 101-108.

5. Barcomes F. 2010. Mordeduras y picaduras de animales. [Internet]. Disponible en: https://www.aeped.es/sites/ default/files/documentos/mordeduras_y_picaduras_de_animales.pdf

6. Beltrán M. 2018. Interacción entre el extracto de Azadirachta indica y el hongo Metarhizium anisopliae para el control biológico de Anopheles albimanus un vector de la malaria. Tesis de Biólogo. Bogotá, Colombia: Univ. de la Salle. 29 p.

7. Boito JP, Da Silva AS, dos Reis JH, Santos DS, Gebert RR, Biazus AH, Baretta D. 2018. Efecto insecticida y repelente del aceite de canela sobre moscas asociadas con el ganado. Revista MVZ Córdoba 23: 6628-6636.

8. Cachago L, Ligña E. 2018. Abundancia y comportamiento alimenticio de Culex quinquefasciatus Say 1823 (Diptera: Culicidae) en la costa norte de Ecuador. Tesis de Maestría. Ecuador: Univ. San Francisco de Quito. 71 p.

9. Cárdenas $\boldsymbol{W} \cdot \mathbf{2 0 1 7}$. Obtención de aceite de semillas de Neem (Azadirachta indica), mediante el método de prensado en frío para determinar su concentración en azadiractina. Tesis de Ingeniero químico. Lambayeque, Perú: Univ. Pedro Ruiz Gallo. 130 p.

10. Castro C, Díaz-Madrigal P. 1998. Pruebas de laboratorio para medir el efecto repelente del NEEM (Azadirachta indica) contra adultos de Aedes aegypti (Diptera: Culicidae). Proyecto de Grado. Costa Rica: Univ. de Costa Rica.

11. Castro MS. 2015. Abatización y métodos de control larvario como medida de prevención del dengue, localidad La
Cruz, Tumbes-Perú, 2013. Manglar 10: 83-91.

12. Celis A, Mendoza CF, Pachón ME. 2009. Uso de extractos vegetales en el manejo integrado de plagas, enfermedades y arvenses: revisión. Temas Agrarios 14: 5-16.

13. Chin J. 2001. El control de las enfermedades transmisibles. Washington: Organización Panamericana de la Salud. 748 p.

14. Chio E, Yang EC. 2008. A bioassay for natural insect repellents. J Asia-Pac Entomol 11: 225-227. doi: 10.1016/ j.aspen.2008.08.002

15. Daza L, Florez N. 2006. Diseño de un repelente para insectos voladores con base en productos naturales. Tesis de Ingeniero de Procesos. Medellín, Colombia: Univ. EAFIT. 133 p.

16. Delgado E, Garcia-Mateo R, YbarraMoncada C, Luna-Morales C, Martinez-Damián T. 2012. Propiedades entomótoxicas de los extractos vegetales de Azadiractha indica, Piper auritum y Petiveria alliacea para el control de Spodoptera exigua Hubner. Rev Chapingo Ser Hortic 18: 55-69.

17. Duong V, Choeung $R$, Gorman $C$, Laurent D, Crabol Y, Mey C, Peng B, et al. 2017. Isolation and full-genome sequences of Japanese encephalitis virus genotype I strains from Cambodian human patients, mosquitoes and pigs. $\mathrm{J}$ Gen Virol 98: 2287-2296. doi: 10.1099/ jgv.0.000892

18. Egunyomi A, Gbadamosi IT, Osiname KO. 2010. Comparative effectiveness of ethnobotanical mosquito repellents used in Ibadan, Nigeria. J Appl Biosci 36: 2383-2388.

19. Escalante MAA, Béjar AAG, de la Rocha RV, Estrada RSG, Fasio AC, Quiroz CC, López JIP. 2004. Contenido de azadiractina A en semillas de NIM (Azadirachta indica A. Juss) colectadas en Sinaloa, México. Rev Fitotecnia Mex 27: 305-311.

20. Esparza-Díaz G, López-Collado J, Villanueva-Jiménez JA, OsorioAcosta F, Otero-Colina G, Camacho- 
Díaz E. 2010. Concentración de azadiractina, efectividad insecticida y fitotoxicidad de cuatro extractos de Azadirachta indica A. Juss. Agrociencia 44: 821-833

21. Fernández R. 2009. El neem: un insecticida fabricado por la naturaleza. [Internet]. Disponible en: https:// www.envio.org.ni/articulo/877

22. García M. 2018. Identificación y control de insectos plagas en el cultivo de maíz criollo en la comuna Sancan. Tesis de Ingeniero Agropecuario. Ecuador: Univ. Estatal del Sur de Manabí. 80 p.

23. Gruber A. 1992. Biología y ecología del árbol de neem (Azadirachta indica A. Juss.) extracción, medición, toxicidad y potencial de crear resistencia. CEIBA $33: 250-256$.

24. Hernández-Castro E, Utrera-Landa V, Villanueva-Jiménez JA, RodríguezLagunes DA, Ojeda-Ramírez MM. 2005. Neem extracts on Aphis nerii behavior and papaya ringspot virus transmission. J Agr U Puerto Rico 89 75-84.

25. Hurtado EA, Loor JDB, Chávez FGA, Ordoñez MVM, Álvarez LG. 2016. Evaluación del extracto acuoso de semilla de neem (Azadirachta indica) como garrapaticida en bovino. Rev Espamciencia 6: 77-80.

26. Kebede Y, Gebre-Michael T, Balkew M. 2010. Laboratorio y evaluación de campo de los aceites de neem (Azadirachta indica A Juss) y Chinaberry (Melia azedarach L) como repelentes contra Phlebotomus orientalis y $P$. bergeroti (Diptera: Psychodidae) en Etiopía. Acta Trop 113: 145-150. doi: 10.1016/j.actatropica.2009.10.009

27. López C. 2015. Evaluación de insecticidas biorracionales para el control de mosquitos Aedes aegypti $(\mathrm{L})=$ Stegomya aegypti en Guasave, Sinaloa. Tesis de Maestría. México: Instituto Politécnico Nacional. $65 \mathrm{p}$.

28. Lorrén F, Ramírez, P, Ramírez, T, Regalado K, Saldarriaga J. 2015. Diseño de una línea de producción para la elaboración de repelente natural a base de aceite de Neem. [Internet]. Disponible en: https://pirhua.udep.edu.pe/ bitstream/handle/11042/2337/ PYT_Infor-me_Final_Repelente $\% 20$ Natural.-pdf?sequence $=1$ \&isAllowed $=y$

29. Mandal S. 2011. Repellent activity of Eucalyptus and Azadirachta indica seed oil against the filarial mosquito Culex quinquefasciatus Say (Diptera: Culicidae) in India. Asian Pacific J Trop Biomed 1: 109-112. doi: 10.1016/S22211691 (11) 60135-4

30. Martínez M, Parra J, Vera M, Vera A. 2016. Parámetros de calidad del aceite de las semillas de Azadirachta indica (neem). Revista CENIC Cienc Quím 47: 70-74.

31. Martínez MA, de Juanes JR. 2006. Viajes internacionales y enfermedades inmunoprevenibles. Vacunas 7: 72-85.

32. Mindiola A. 2019. Proceso de elaboración del bioinsecticida botánico «Apichi» mediante la utilización de extractos vegetales con propiedades plaguicidas. Tesis de Ingeniero Agrónomo. Ecuador: Univ. Técnica de Babahoyo. $21 \mathrm{p}$.

33. Morales D. 2019. Evaluación del efecto repelente de dos concentraciones del extracto de Neem (Azadirachta indi$c a$ ) en moscas hematófagas de bovinos, administrado por vía tópica. Tesis de Médico Veterinario. Guatemala: Univ. de San Carlos de Guatemala. 56 p.

34. Morán C. 2018. Uso de bioinsecticida a base de neem Azadirachta indica para el manejo de saltahoja en agroecosistema de caña de azúcar, Guayas, Ecuador. Manglar 14: 73-83.

35. Morgan J. 2012. Control of biting insects on pigs. Department of Primary Industries, Australia. [Internet]. Available in: https://www.dpi.nsw.gov.au/_data/ assets/pdf_file/0006/425832/Control-ofbiting-insects-on-pigs.pdf

36. Naseem S, Malik MF, Muni, T. 2016. Mosquito management: a review. J Entomol Zool Estudies 4: 73-79.

37. Novo RJ, Viglianco A, Nassetta M. 1997. Actividad repelente de diferentes extractos vegetales sobre Tribolium 
casta-neum (Herbst). Agriscientia 14: 31-36. 10.31047/1668.298x.v14.n0.2524

38. Olaya JM, Méndez J. 2003. Guía de plantas y productos medicinales. Bogotá, Colombia: Siglo del Hombre. 32 p.

39. Pérez C, González L, Colón A, Morello C, Mujica V, Martínez A. 2009. Evaluación comparativa de los rendimientos obtenidos mediante el proceso de extracción en aceites vegetales a partir de semillas oleaginosas. Anales Univ Metropolitana 9: 181-206.

40. Ramírez-Suárez T, Ramírez-Calderón I. 2018. Obtención y propuesta de producción por lotes de un repelente natural a base de aceite de neem. Tesis de Ingeniero Industrial y de Sistemas. Piura, Perú. Univ. de Piura. 249 p.

41. Regnault-Roger C. 2012. Trends for commercialization of biocontrol agent (biopesticide) products. In: Plant defence: biological control. Springer. p 139-160.

42. Rodríguez, M, Alcaraz L, Real S. 2012. Procedimientos para la extracción de aceites esenciales en plantas aromáticas. Centro de Investigaciones Biológicas del Noroeste. Baja California, México: $38 \mathrm{p}$.

43. Roldán J, Morales R, Otiniano G. 2015. Efecto repelente del aceite del endospermo de Ricinus communis (Euphorbiaceae) en Culex quinquefasciatus (Diptera: Culicidae), bajo condiciones experimentales. Rebiol 35: 82-90.

44. Romero C, Vargas M. 2005. Extracción de aceite de la semilla de neem (Azadirachta indica). Ciencia 13: 464-474.

45. Sanabria SR, Ramírez M. 2013. Evaluación del efecto insecticida y repelente del polvo de Chenopodium ambrosioides sobre adultos de Callosobruchus maculatus F. (Coleoptera, Bruchidae) en semillas de poroto (Vigna unguiculata). Inv Agraria 11: 36-39.

46. Santamaría E, Cabrera OL, Zipa Y, Pardo RH. 2012. Eficacia en campo- de un repelente a base de para-mentano3, 8-diol y aceite de limonaria contra Culicoides pachymerus (Diptera: Ceratopogonidae) en Colombia. Biomédica 32: 457-460.

47. Shooshtari MB, Kashani HH, Heidari $S$, Ghal R. 2013. Comparative mosquito repellent efficacy of alcoholic extracts and essential oils of different plants against Anopheles Stephensi. Afr J Pharm Pharmaco 7: 310-314.

48. Tabassum S, Sharadha R, Siddappa MR, Abbulu K. 2018. A research on mosquito repellent and larvicidal activity of NEMCAM (Azadirachta indica and Cinnamomum camphora). J Pharmacog Phytochem 7: 2234-2238.

49. Tesfaye B, Tefera T. 2017. Extraction of essential oil from neem seed by using soxhlet extraction methods. Int $\mathrm{J} \mathrm{Adv}$ Engin Manag Sci 3: 646-650.

50. Thorsell W, Mikiver A, Malm E, Mikiver M. 1989. Fly repellents from extracts of Mentha spicata x crispatastudies on Protophormia terraenovae. Entomol Tidskrift 110:109-112.

51. Vargas MV. 1998. El mosquito: un enemigo peligroso: biología, control e importancia en la salud humana (Diptera: Culicidae). San José, Costa Riva: Univ. de Costa Rica. 264 p.

52. Vega-Jarquín C. 2016. Identificación de metabolitos bioactivos de Neem (Azadirachta indica Adr. Juss.). La Calera 14: 60-66. doi: 10.5377/calera.v14i23.2659

53. [WHO] World Health Organization. 2009. Guidelines for efficacy testing of mosquito repellents for human skin: Geneva: World Health. [Internet]. Available in: https://apps.who.int/iris/bitstre$\mathrm{am} / \mathrm{handle} / 10665 / 70072 / \mathrm{WHO}$ HTM_NTD_WHOPES_2009.4en g.pdf; j s e s s i on i d $=25$ BD56263DE7A5FDD07-BFC84E740A6C5? sequence $=1$ 